\title{
Readability Evaluation of Drug Package Inserts for Children with Asthma in China
}

\author{
Xiaowen Xing ${ }^{1}$, Yibo $\mathrm{Wu}^{2 *}$, Zeyu Jiang ${ }^{1}$, Cunyi $\mathrm{Ku}^{1}$ \\ ${ }^{1}$ School of Pharmacy, Shandong University, Jinan, Shandong, China; ${ }^{2}$ Department of Pharmacy Administration \\ and Clinical Pharmacy, School of Pharmaceutical Sciences, Peking University, Beijing, China.
}

${ }^{*}$ Address for correspondence: Yibo Wu, Department of Pharmacy Administration and Clinical Pharmacy, School of Pharmaceutical Sciences, Peking University, Xueyuan Road NO.38, Haidian, Beijing100191, China. E-mail: bjmuwuyibo@outlook.com

Received May 28, 2019; Accepted August 17, 2019

In order to understand the current situation of the readability of drug package inserts in China, in this paper, the readability evaluation and analysis of the drug package inserts for children with asthma are carried out. Based on the results obtained, reasonable suggestions are put forward. The results show that the asthma drug package inserts for children in China are easy to read. However, there are differences in the readability of different types of drugs. For example, Chinese medicines were easy to read but not clear, and western medicines were difficult to read and understand; package inserts of foreign enterprises were more challenging to read than that of state-owned enterprises; prescription drugs were more difficult than over-the-counter drugs to read; large difference in that readability of each dosage form. Therefore, our country's drug package inserts should perfect the content, enhance the scientificity and readability; expand the range of variables and develop more targeted formulas; simplify the content and design the package inserts for the patient; pay attention to designing of information and improving the readability of text.

Key Words: Drug package insert; Readability; Safe drug use

\section{INTRODUCTION}

The 19th CPC National Congress clearly stated that "the implementation of a healthy China strategy" and "safeguarding the safety of medicines" is an essential part of it. As a statutory document that informs important information about drugs, the drug package inserts are an important basis for the selection of drugs and play a significant role in guiding patients to use drugs. ${ }^{1,2}$ It differs from the general text in its higher normative and scientific and more medical terms. However, only $21.57 \%$ of the residents were able to "fully understand" the contents of the drug package inserts, and "not completely understand" was the main reason, ${ }^{3}$ this suggests that residents' understanding of the drug package inserts may be related to their readability. ${ }^{4-7}$ Readability refers to the text shape, the content itself is read by the reader and the degree of accurate understanding. ${ }^{8}$ This topic intends to evaluate and analyze the readability of the manual on the common children's asthma drugs in the market as examples. Based on the results, it provides reasonable suggestions to enhance the readability of the Chinese package insert.

\section{MATERIALS AND METHODS}

\section{Materials}

Text Source and Search Strategy

Select "Clove Garden Medication Assistant - Drug Manual," “360
Health", "39 Health Network - Drug Channel - Drugs" and "Seek Medical Network - Disease Search" as the database. These are the large scale health websites in China, and the drug websites under them have many kinds of drugs and comprehensive information. Search indications for "pediatric asthma". Of the drugs, obtained $11,54,61$ and 34 results, and find their instructions.

\section{Sample selection and exclusion principles}

Out of the initial 160 search results, 6 were excluded from non-pediatric use, 95 were identical drugs, and 59 different drugs were obtained. 263 instructions were retrieved and 202 identical or similar results were excluded. A total of 61 valid results were obtained and a database was established. This is an open access journal, and articles are distributed under the terms of
the Creative Commons Attribution-NonCommercial-ShareAlike 4.0 License,
which allows others to remix, tweak, and build upon the work non-commercially, as
long as appropriate credit is given and the new creations are licensed under
the identical terms.

For reprints contact: weda-h@ weda-h.org

How to cite this article: Xing XW, Jiang $\mathrm{ZY}, \mathrm{Ku} \mathrm{CY}, \mathrm{Wu} \mathrm{YB}$. Readability Evaluation of Drug Package Inserts for Children with Asthma in China. J ADV HEALTH 2019; 1(3): 190-196. 


\section{Method}

\section{Formula selection and introduction}

In this article, the easy-to-read formulas for children's asthma medicines in China were measured by two easy-to-read formulas: "comprehension score(CS)" and "occlusion score(OS)" proposed by Mr. Yang Xiaotong. ${ }^{9}$ He proposed the easy-to-read formula with the newspaper as a sample, and was subsequently applied to the news readability measurement, ${ }^{10}$ the readability measurement of the consent form, ${ }^{11}$ etc., indicating that the formula can be applied to medical texts, and can be used for the readability measurement of the Chinese medicine specification.

(1)Comprehension score formula:

$$
\mathrm{X}_{1}=3.5921+0.8826 \mathrm{X}_{2}-0.0179 \mathrm{X}_{3}
$$

Among them: X1: comprehension score; X2: count one to ten rate, which means that the Chinese text contains the ratio of the total number of strokes in one to ten drawing words; X3: special word rate, refers to the proper nouns in Chinese text, such as the name, place name, agency name, etc.

(2) Occlusion score formula:

$$
\mathrm{X}_{4}=0.3344+1.4593 \mathrm{X}_{5}-0.168 \mathrm{X}_{6}
$$

Among them: X4: occlusion score; X5: completely symmetrical word rate, completely symmetrical words are words that are completely symmetric in Chinese words, such as "medium", "flat", "single", etc., and full symmetric word rate means the ratio of complete symmetry and total number of words; X6: The rate of difficult words, mainly referring to professional terms in the field of health care.

Table 1. Chinese difficulty level distribution table

\begin{tabular}{cccccc}
\hline \multirow{2}{*}{ Readability measurement } & \multicolumn{5}{c}{ Degree of difficulty } \\
\cline { 2 - 6 } & Very easy & Easy & Medium & Difficult & Very difficult \\
\hline CS & $4.7029-5.0000$ & $4.3202-4.7028$ & $3.9827-4.3201$ & $3.6002-3.9828$ & $0.0000-3.6001$ \\
OS & $0.6780-1.0000$ & $0.6041-0.6779$ & $0.5390-0.6040$ & $0.4651-0.5389$ & $0.0000-0.4650$ \\
\hline
\end{tabular}

\section{Calculation method}

For example: 肺立咳合剂(A drug for treating asthma in children) 【功能主治】中医: 清热解毒, 镇咳祛痰。用于小儿痰热犯肺 所引起的咳嗽痰黄, 支气管哮喘, 气管炎见上述证候者。 (Functional indication: theory of traditional Chinese medicine: heat and detoxification, antitussive expectorant. Used for cough, phlegm yellow, bronchial asthma and tracheitis caused by phlegm fever in children.)This part explains the functions of traditional Chinese medicine, and then explains the disease of the attending from western medicine. Relatively easy to understand so selected as examples.

In this description, there are 44 Chinese characters; one to ten painted words 33; special words 0 ; symmetrical words 10 ;

$\mathrm{X}_{1}(\mathrm{CS})=3.5921+0.8826 \mathrm{X}_{2}$ (one to ten rate) $-0.0179 \mathrm{X}_{3}$ (special word rate)

$\mathrm{X}_{1}=3.5921+0.8826 \times 33 / 44-0.0179 \times 0 / 44=4.2541$ (medium)

$\mathrm{X}_{4}(\mathrm{OS})=0.3344+1.4593 \mathrm{X}_{5}$ (full symmetrical word rate)-

$0.168 \mathrm{X}_{6}$ (hard word rate)

$\mathrm{X}_{4}=0.3344+1.4593 \times 10 / 44-0.168 \times 12 / 44=0.6202$ (easy)

\section{Research content}

This article analyzes the specifications of children's asthma medicines in China from five aspects: (1)Easy-to-read comparison within the specification, including names, components, adverse reactions, toxicological studies, children medications, functional indications, specifications, taboos, elderly patients drug use, adaptability, traits, pharmaceutics, pharmacology and toxicology, drug overdose, drug interactions, usage and dosage, medication for pregnant and lactating women, storage, precautions, packaging, expiration date, approval number, manufacturing company. The contents of the item, because of the contents of the package, validity period, approval number, and production company's "Manual of Drug Labeling and Labeling," do not have any difference. This article does not make statistics. (2)Comparison of easy readability of Chinese and Western medicine instructions.
(3)Comparison of the readability of the manuals of state-owned enterprises and foreign companies. (4)Comparison of the readability of prescription and non-prescription instructions. (5)Comparable readability of different formulations including freeze-dried powder, aerosol, decoction, suppository, granule, oral solution, powder, pill, mixture, solution, capsule, aerosol, injection, and tablet Different dosage forms.

\section{RESULTS AND DUSCUSSION}

\section{Comparison of various parts of the drug product specification}

The CS of the Pediatric Asthma Drugs Manual in China was $49.61 \%$ for "easy" and "medium", $0.39 \%$ for "difficult" and "very difficult", and "very easy" did not appear. In general, the manual was more readable. China's children's asthma kits accounted for more than $80.00 \%$ of the total bill rate, and no particular words such as the State Food and Drug Administration appeared in the manual. Therefore, the overall drug listing was more comfortable to read from the point of CS.

The overall OS of children's asthma medicines in China was $32.99 \%$, "easy" $16.88 \%$, "medium" $14.30 \%$, "difficult" $17.78 \%$, and "very difficult" $18.04 \%$, but overall it was easier to read. There were differences in the legibility of various contents. Among them, children's medication, medications for the elderly, contraindications, usage and dosage were the most readable, and the proportions of "very easy" were $78.57 \%, 76.92 \%, 65.38 \%$, and $63.93 \%$, respectively. The most readable content was about usage, usage, and taboos, which studies indicated were among the top concerns of drug users, ${ }^{12-15}$ the usage and usage part of the difficult terms such as medical terms was almost 0 , the taboo part of the average symmetry rate was $34.11 \%$, so it was the easiest to read. The more readable was the name of the drug, its function indications, and its traits. The sum of its "very easy" and "easy" proportions was $66.17 \%, 66.67 \%$, and $74.14 \%$, respectively. Most Western drug names contained difficult words, such as "isoproterenol hydrochloride"; rare diseases such as "a stroke bronchitis" 


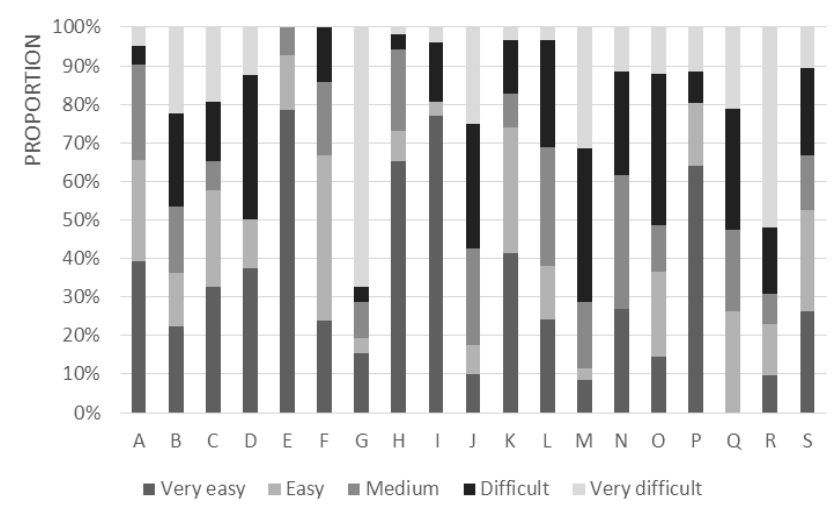

Figure 1. Understanding scores of various contents of the manual A:name B:components C:adverse reactions D:toxicological study E:children's medication F:functional indication G:specifications H:taboos I:elderly patients drug use J:adaptability K:traits L:pharmaceutics M:pharmacology and toxicology $\mathrm{N}$ :drug overdose O:drug interaction P:usage and dosage Q:medication for pregnant and lactating woman R:storage S:precautions

sometimes appeared in functional indications; chemical terms such as "mixed" appear in the traits. "Suspension", which had a low frequency of occurrence of such words and asymmetry rate of $20 \%$ to $30 \%$, was easier to read. The proportions of "very difficult" in specifications and storage were the largest, which were $64.46 \%$ and $51.92 \%$, respectively. The odds and symmetry ratios in specifications and storage were mostly $0.00 \%$, and the calculation result of the OS formula was 0.3344 , which was "very difficult". However, in reality, this part was extremely brief, and it was mostly a more readable expression such as "seal" and "preservation from light." This error may be due to the fact that the OS formula had fewer variables and its applicability to some medical texts was not tall. Pharmacological studies, drug interactions, pharmacology and toxicology, and indications were relatively difficult to read. The sums of "difficult" and "very difficult" were 50.00\%, 51.40\%, $71.43 \%$, and $57.50 \%$, respectively. These three kinds of medical terms, such as "beta receptor agonist" and other difficult words appeared more, mostly about $40 \%$, and the symmetry rate was low, mostly about $12 \%$, increasing the difficulty of reading. The

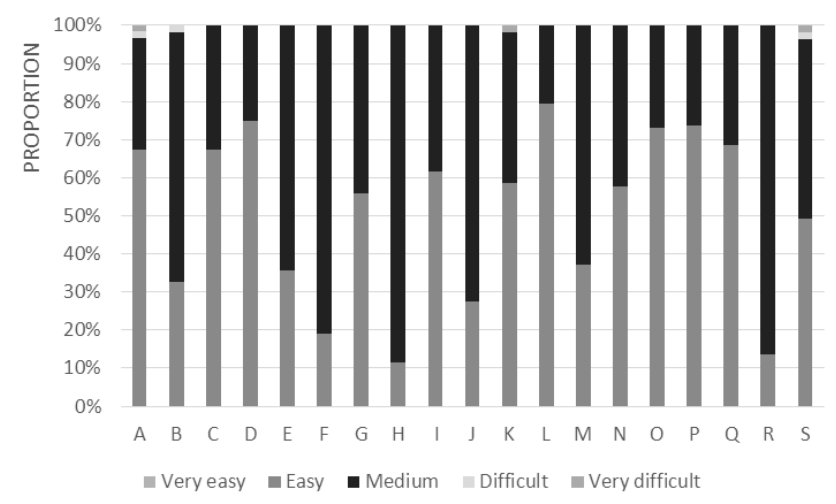

Figure 2. OS for various contents of the specification

A:name B:components C:adverse reactions D:toxicological study E:children's medication F:functional indication G:specifications H:taboos I:elderly patients drug use J:adaptability K:traits L:pharmaceutics $\mathrm{M}$ :pharmacology and toxicology $\mathrm{N}$ :drug overdose O:drug interaction P:usage and dosage Q:medication for pregnant and lactating woman R:storage S:precautions proportions of ingredients, $\mathrm{AD}$, overdosage, medications for women and children, and precautions were not much different. The readability was "medium". This may be because the content of this part was lower than that of pharmacology and toxicology, and thus difficult words appeared. Less, the language was more popular.

Comparison of the readability of package leaflets of traditional Chinese medicines and Western medicines

Traditional Chinese medicines were used under the guidance of Chinese medicine theory, while western medicines were used under the guidance of modern medical theory, and they both had an irreplaceable role in the treatment process. With the CS, the proportion of the Chinese medicine instructions evaluated as "easy" was $66.67 \%$; and with the OS, the sum of the proportion of evaluation as "easy" and "very easy" was $83.33 \%$. Therefore, the readability of Chinese medicine instructions was relatively easy as a whole. However, there were $37.21 \%$ of western medicine package leaflets evaluated as "easy" and $62.79 \%$ evaluated as "medium" based on the CS; and with OS, the proportion of "difficult" was $30.23 \%$, some package leaflets even were assessed as "very difficult", which indicated that the readability of western medicine instructions was evidently more difficult. Firstly, in the Chinese medicine instructions, such as pediatric heat relieving cough pills, the main information of the package leaflets like dosage, contraindications, adverse drug reactions and precautions were too simple to be understood, some even were not clear yet. Moreover, the omission of drug interactions, pharmacology and toxicology, drug overdose and pharmacokinetics led to low readability. Secondly, the content of western medicine instructions was more professional than that of Chinese medicine instructions. Numerous experiments and academic terms were required to explain the mechanism drug actions clearly, resulting in an increase in the number of strokes and difficult words. For instance, the proportion of difficult words in the pharmacology and toxicology about Methylprednisolone Tablets up to $59.38 \%$, while the overall difficulty of the package leaflets was only $41.03 \%$. The two reasons mentioned above had caused the situation that "Chinese medicine is unclear and western medicine is difficult to understand".

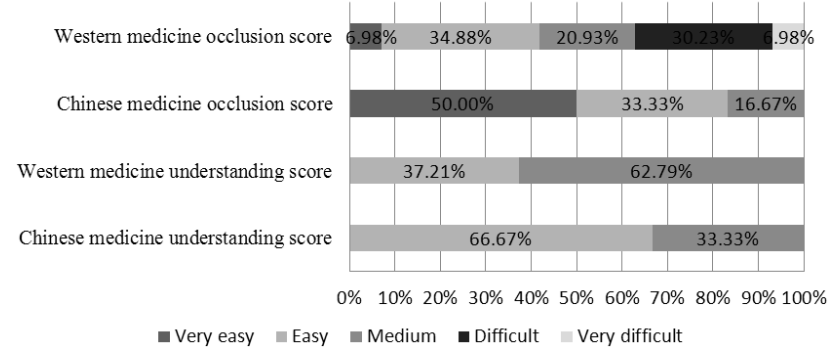

Figure 3. Comparison of the readability of Chinese and western medicine instructions

Comparison of the readability of package leaflets of stateowned medicines and foreign enterprise medicine

In the statistical package leaflets, the Chinese company drug instructions accounted for $90.16 \%$, and the foreign company drug instructions accounted for $9.84 \%$. The CS of foreign company medicine package leaflets were all "easy", and the "easy" of the 
state-owned medicines was $34.55 \%$; with OS, there was no "very easy" and "easy" for the package leaflets of foreign enterprise medicines, while that of state-owned enterprises were varied and "very easy" and "easy" accounted for $23.64 \%$ and $36.36 \%$ each, indicating that the readability of the state-owned medicine package leaflets, was uneven and that the foreign enterprise medicine package leaflets were more difficult to read than state-owned medicine package leaflets. The main reasons for this situation were as follows: (1)State-owned medicines contained many types of drugs, including traditional Chinese medicines, western medicines, and various dosage forms. The depth of research in different aspects was not uniform, as a result of which, the readability of package leaflets was uneven. (2)Foreign companies' drugs were all western medicines. The content of ingredients, toxicology studies, pharmacokinetics, and drug interactions, which including many complicated words such as chemical names, medical nomenclature, was in-depth. The symmetric word rate was low, mostly about $14.50 \%$, so after the calculation of the occlusive fraction formula, the results were most difficult to read.

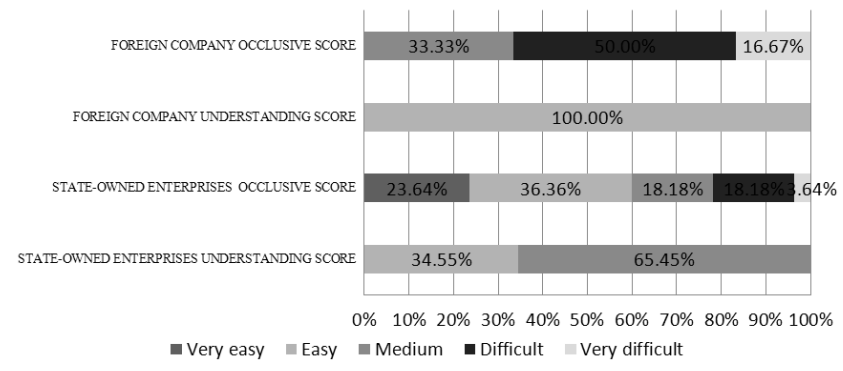

Figure 4. Comparison of the readability of package leaflets of stateowned medicines and foreign enterprise medicine

\section{Comparison of the package leaflets of prescription and non- prescription medicines}

Overall, the readability of the two types of drugs presented a situation that "prescription drug package leaflets were more difficult to read than that of non-prescription drugs." The results of CS indicated that the readability of the package leaflets concentrated on "easy" and "medium"; while the results of OS were more diverse, especially the readability of the prescription package leaflets ranged from "very easy" to "very difficult."

From the results of CS, the proportion of "easy" and "medium" in prescription and non-prescription medicines were roughly the opposite, namely, $43.14 \%$ of prescription-medicine instructions were "easy" and $56.86 \%$ were "medium"; $60.00 \%$ of nonprescription medicine instructions were "easy", and $40.00 \%$ was"medium". From the results of OS, the readability of the non-prescription medicine package leaflets that were below the "medium" difficulty, namely, easy for general reading comprehension, accounted for $80.00 \%$; that of the prescription medicine instructions which were below the "medium" difficulty accounted for $49.02 \%$, the "medium" difficulty was $19.61 \%$, and the above "medium" difficulty was $31.37 \%$.

The results from CS and OS both indicated that prescription medicine package leaflets were more difficult to read than non-prescription medicine instructions. From CS, because there were no special words in the two types of instructions, the degree of readability depended mainly on the rate of one to ten rate. Non-prescription medicine instructions contained less information, the total number of words were mostly concentrated on 400 , one to ten strokes' words were 350 or so, which means one to ten rates were higher, demonstrating that the package leaflets were easier to read. However, compared with non-prescription medicines, prescription medicine package leaflets, which added instructions for children, the elderly, pregnant women and breastfeeding women, were more comprehensive. Therefore, the total number of words and multi-stroke characters increased significantly, leading to a decrease in one to ten rate and low readability. From the perspective of OS, the rate of difficult words was a determining factor in the readability of the two types of package leaflets. Compared with non-prescription medicine package leaflets, more specialized content such as pharmacology and toxicology and overdose were contained in the prescription medicine package leaflets, giving rise to the increasing number of difficult words and low readability.

In addition, differences in the readability of the package leaflets may also be related to the differences in the use of prescription and non-prescription drugs. Non-prescription drugs can be purchased and used without the guidance of a doctor. Therefore, there were clear requirements for the design of non-prescription medicine package leaflets in China. For example, Article 5 of the Drug specification and labeling regulations states that "The written description of drug instructions and labels should be scientific, standardized and accurate. The non-prescription drug instructions should also use easily understandable written descriptions so that patients can make their judgments, choices, and uses", making the non-prescription medicine package leaflets even easier to read. Prescription drugs were available only by prescription, and doctors would also give medication guidance to patients, which to a certain extent lowering the requirement for patients to understand prescription medicine package leaflets.

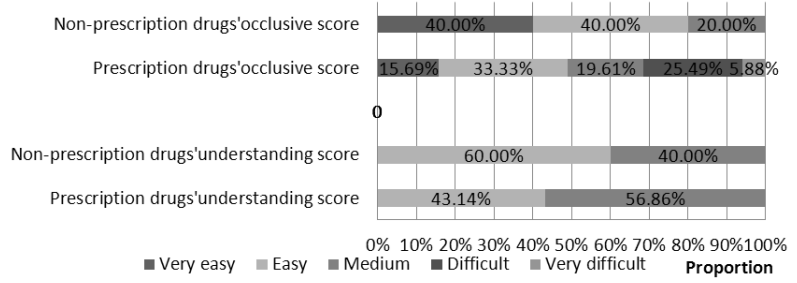

Figure 5. Proportion of readability of prescription and non-prescription drugs

\section{Comparison of different dosage forms}

Overall, each dosage form's ratio of easy-to-read package leaflets (the readability was "easy" and "easy") to difficult-to-read package leaflets (the readability was "medium", "difficult", and "very difficult") was close to $1: 1$. The results of CS indicated that $45.90 \%$ of the package leaflets were "easy" and $54.10 \%$ were "medium"; the results from the OS were distributed in every reading difficulty, $19.67 \%$ of the package leaflets were "very easy", $34.43 \%$ were "easy", $19.67 \%$ were "medium", $21.31 \%$ were "difficult" and 4.92\% were "very difficult".

From the results of CS, the package leaflets of soft extract, granules, solutions, injections, and tablets had a higher proportion of "medium" difficulty, indicating that these dosage forms were more difficult to understand. This may be due to the fact that the content of these dosage forms was more comprehensive, the total number of words and multi-stroke characters increased, one to ten rate reduced, leading to low readability. From the results of OS, the 
"medium", "difficult" and "very difficult" package leaflets of the freeze-dried powder, powder aerosol, capsules, aerosols, and tablets accounted for more than $50 \%$, demonstrating the package leaflets of these dosage forms was difficult to read. It may be related to the increase of difficult words, for example, there were many specialized words such as "fluticasone propionate" and "salbutamol" appeared in the package leaflet of salmeterol xinafoate and fluticasone propionate powder for inhalation, which were difficult for patients to understand. The combined results of
CS and OS showed that the package leaflets of tablet were the most difficult to read, which may be due to $80 \%$ of the tablets were prescription drugs, the content of the package leaflets was more in-depth and comprehensive, the specialized terms and difficult words increased, one to ten rate and symmetrical words decreased, thus the legibility of the package leaflets reduced. Additionally, there were all kinds of tablet drugs, and the readability level distributed widely, therefore, the OS of tablet drugs were distributed in every reading difficulty.

Table 2. Comparison of different dosage forms

\begin{tabular}{|c|c|c|c|c|c|c|c|c|c|c|}
\hline \multirow{2}{*}{ Dosage form } & \multicolumn{5}{|c|}{ Understanding score } & \multicolumn{5}{|c|}{ OS } \\
\hline & Very easy & Easy & Medium & Difficult & Very difficult & Very easy & Easy & Medium & Difficult & Very difficult \\
\hline Freeze-dried powder & $0.00 \%$ & $100.00 \%$ & $0.00 \%$ & $0.00 \%$ & $0.00 \%$ & $0.00 \%$ & $0.00 \%$ & $100.00 \%$ & $0.00 \%$ & $0.00 \%$ \\
\hline Powder aerosol & $0.00 \%$ & $100.00 \%$ & $0.00 \%$ & $0.00 \%$ & $0.00 \%$ & $0.00 \%$ & $0.00 \%$ & $100.00 \%$ & $0.00 \%$ & $0.00 \%$ \\
\hline Soft extract & $0.00 \%$ & $0.00 \%$ & $100.00 \%$ & $0.00 \%$ & $0.00 \%$ & $100.00 \%$ & $0.00 \%$ & $0.00 \%$ & $0.00 \%$ & $0.00 \%$ \\
\hline Suppository & $0.00 \%$ & $100.00 \%$ & $0.00 \%$ & $0.00 \%$ & $0.00 \%$ & $0.00 \%$ & $100.00 \%$ & $0.00 \%$ & $0.00 \%$ & $0.00 \%$ \\
\hline Granula & $0.00 \%$ & $0.00 \%$ & $100.00 \%$ & $0.00 \%$ & $0.00 \%$ & $100.00 \%$ & $0.00 \%$ & $0.00 \%$ & $0.00 \%$ & $0.00 \%$ \\
\hline Oral liquid & $0.00 \%$ & $50.00 \%$ & $50.00 \%$ & $0.00 \%$ & $0.00 \%$ & $0.00 \%$ & $50.00 \%$ & $50.00 \%$ & $0.00 \%$ & $0.00 \%$ \\
\hline Powder & $0.00 \%$ & $100.00 \%$ & $0.00 \%$ & $0.00 \%$ & $0.00 \%$ & $50.00 \%$ & $50.00 \%$ & $0.00 \%$ & $0.00 \%$ & $0.00 \%$ \\
\hline Pills & $0.00 \%$ & $50.00 \%$ & $50.00 \%$ & $0.00 \%$ & $0.00 \%$ & $50.00 \%$ & $50.00 \%$ & $0.00 \%$ & $0.00 \%$ & $0.00 \%$ \\
\hline Mixture & $0.00 \%$ & $100.00 \%$ & $0.00 \%$ & $0.00 \%$ & $0.00 \%$ & $66.67 \%$ & $33.33 \%$ & $0.00 \%$ & $0.00 \%$ & $0.00 \%$ \\
\hline Solution & $0.00 \%$ & $33.33 \%$ & $66.67 \%$ & $0.00 \%$ & $0.00 \%$ & $33.33 \%$ & $33.33 \%$ & $33.33 \%$ & $0.00 \%$ & $0.00 \%$ \\
\hline Capsule & $0.00 \%$ & $60.00 \%$ & $40.00 \%$ & $0.00 \%$ & $0.00 \%$ & $0.00 \%$ & $40.00 \%$ & $40.00 \%$ & $20.00 \%$ & $0.00 \%$ \\
\hline Aerosol & $0.00 \%$ & $80.00 \%$ & $20.00 \%$ & $0.00 \%$ & $0.00 \%$ & $0.00 \%$ & $0.00 \%$ & $40.00 \%$ & $60.00 \%$ & $0.00 \%$ \\
\hline Injection & $0.00 \%$ & $0.00 \%$ & $100.00 \%$ & $0.00 \%$ & $0.00 \%$ & $0.00 \%$ & $63.64 \%$ & $9.09 \%$ & $18.18 \%$ & $9.09 \%$ \\
\hline Tablet & $0.00 \%$ & $45.45 \%$ & $54.55 \%$ & $0.00 \%$ & $0.00 \%$ & $18.18 \%$ & $27.27 \%$ & $13.64 \%$ & $31.82 \%$ & $9.09 \%$ \\
\hline
\end{tabular}

\section{CONCLUSION AND PROSPECT}

\section{Perfect the content, enhance the scientificity and readability}

Compared with foreign drug package inserts, there was a lack of information in our country's drug package inserts. ${ }^{16,17}$ Among them, the lack of information of the domestic drug package inserts was higher than that of the foreign drug package inserts; traditional Chinese medicine and foreign medicine was the same as them. For example, the content of the drug mechanism in the domestic manual was less involved or insufficient. In addition, there was a lack of information about medication information. Therefore, the contents of the drug package inserts should be replenished, updated and perfected in time to the drug research, so as to promote rational drug use and reduce the risk of drug use due to lack of information. ${ }^{18}$ At the same time, it was necessary to weigh the scientificity and readability of drug instruction in general. Scientificity the primary condition of medical instructions, which was an important prerequisite for ensuring that doctors and patients get the correct medication information. Therefore, the design of drug package inserts should follow the principle of scientific priority, accurate wording, complete data and comprehensive content science. ${ }^{19}$ In the context of scientificity, we should consider the readability of the drug package inserts. For example, by adjusting the layout of the specification structure, ${ }^{20}$ designing the information, designing the instructions for the patients and so on to improve the readability of the manual, the reading efficiency of the patient, and finally reach the organic harmonics of the scientificity and readability of the drug package inserts.

\section{Expand the range of variables and develop more targeted formulas}

In theory, there was almost no special word in the drug package inserts, which led to that the understanding score contains only one variable of "one to ten rate", which was less accurate than the OS of two variables. In terms of the actual measurement results, for example, in the comparison between prescription and non-prescription manual, the evaluation results of understanding score only include "easy" and "medium", while the results of the OS were distributed in all difficulty ranges. It can be seen from this that the distinction and accuracy of the OS was higher than the understanding score, which was, in a certain degree, the more variables involved, the higher the accuracy of the formula. Therefore, the researchers can increase the accuracy and distinction of the reading formula by increasing the length of the sentence, the frequency of the words, and the technical terms of the words. In addition, the formula used in this paper was widely applicable to medical text, but its accuracy was reduced when it was applied to the specific text of drug instruction. This showed that the wider the application scope of the readability formula, the more difficult it was to ensure its accuracy. Therefore, in order to improve the situation of low accuracy and low pertinence of readability formula, it was necessary to strengthen the development of precise and readability formulae, such as 
developing formulas for specific texts such as drug specification, informed consent and patient education materials, so as to make scientific and accurate evaluation of all kinds of medical texts scientifically and accurately.

In addition, with the development of the future society, the content of the package inserts will cover more aspects, ${ }^{21}$ and the number of words in the specification will increase explosively, which is particularly prominent in the preparation of traditional Chinese medicine. Moreover, children's understanding of the text is much less than adults, so children package inserts should be simple, attractive, and easy to understand. For the easy-to-child drug description manual evaluation model, the new formula can be established based on the original legibility formula. The formula variables mainly include "counting one to ten rate", "total number of words" , "vitality", and "difficult word rate". The difficult word rate should be the main reason for determining whether the paragraph is legible. In order to ensure the scientific nature of the drug manual, the difficult words in the manual can hardly be modified, but the target population of the manual is only the children, and the difficult word selection can make compromises, that is, difficult words can be designed into short sentences that are easy for children to understand, or pictures can be used to replace some sentences in package inserts to increase the readability of drug instructions. ${ }^{22}$

\section{Simplify the content and design the package inserts for the patient}

Drug package inserts for information was various and had too many technical words in the part project such as pharmacology and toxicology, pharmacokinetics, which caused the legibility overall was more difficult. ${ }^{23}$ In addition, it also exposed the manual reading difficulties caused by specialized. A lot of foreign countries put it in a medicine box for the patient or at the same time both the medical staff and the patient. However, the domestic manual was mainly aimed at the medical staff, which caused the situation that the patient was unable to understand the manual. ${ }^{24}$ The contents of the manual should be related to the education level and the actual application of the patients, ${ }^{25}$ and the items concerned should be easily understood and read by everyone who requires. ${ }^{26}$ For example, in addition to the general knowledge of drug use, medical workers pay more attention to the pharmacokinetics of medicine, but ordinary patients pay more attention to the general information such as the use and storage of drugs. ${ }^{27,28}$ It was imperative to design a manual for patients, on the one hand, to eliminate some of the professional content and retain more attention of patients, mainly including drug name, indication, contraindication, drug use, storage, date, and drug overdose information, etc. On the other hand, information about safety and effectiveness in the manual should be communicated to patients in simple and understandable words. ${ }^{29-31}$ At present, there was a large gap between the design of domestic package inserts and the developed countries. We can learn from the experience of the United States, Australia and other countries, and combine with our current situation to serve our country's medical and health services better. $^{32}$

\section{Pay attention to designing of information and improving the readability of text}

Information design was through the means of art and science of information processing, making it more effective, quickly accepted by the people, ${ }^{33}$ which played a vital role in improving on the reader experience and auxiliary popular science propaganda. Information design should include the following: (1)Label design. For example, the key information in the package inserts, especially the information about the adverse reactions and the risk of drug use, can attract the patients' visual attention by designing colorful titles ${ }^{34,35}$ and special symbols such as " " and " $\Delta$ ". The key text can cause the attention of the patient by adding a black box to the text, adjusting the font size or tilting the font. (2)Information modularization. In order to avoid the difficulty of reading the large dense text, it should be divided into multiple text blocks. The package inserts can be used to enhance the visual perception through proper retention, spacing lines, and use of tables. (3)Information pictorialization. For some conceptual text, such as the use of special drugs, we can improve its readability by adding a diagram. ${ }^{36-40}$ (4) Add QR code. Display more complicated drug use instructions in the form of audio or video.

\section{Conflict of interest}

There are no conflicts of interest.

\section{Acknowledgement}

This work was supported by the China Association for Science and Technology under (Grant No. kxyjskpxm2019024 and kxyjskpxm 2019055).

\section{REFERENCES}

1. Sawalha A, Sweileh W, Zyoud S, Jabi S. Comparative analysis of patient package inserts of local and imported anti-infective agents in palestine. Libyan J Med 2008; 3(4): 181-185.

2. Al-Aqeel SA. Evaluation of medication package inserts in Saudi Arabia. Drug Healthc Patient Saf 2012; 4: 33-8.

3. Wan HS. Investigation and Analysis of Residents' Cognition on Drug Instructions in Huancheng Community, Binhai County, Jiangsu Province. Chinese Pharmacy 2017; 28(24): 3333-6. (in Chinese).

4. Masland MC, Kang SH, Ma Y. Association between limited English proficiency and understanding prescription labels among five ethnic groups in California. Ethn Health 2011; 16(2): 125-44.

5. Davis TC, Wolf MS, Bass PF 3rd, Middlebrooks M, Kennen E, Baker DW, Bennett CL, Durazo-Arvizu R, Bocchini A, Savory S, Parker RM. Low literacy impairs comprehension of prescription drug warning labels. J Gen Intern Med 2006; 21(8): 57-62.

6. Davis TC, Michielutte R, Askov EN. Practical assessment of adult literacy in health care. Health Educ Behav 1998; 25(5): 613-24.

7. Wolf MS, Davis TC, Tilson HH, Bass PF 3rd, Parker RM. Misunderstanding of prescription drug warning labels among patients with low literacy. Am J Health Syst Pharm 2006; 63(11): 1048-55.

8. Cao Y, Zhou SM. Progress of Research on Health Information Readability. China Health Education 2013; 29(7): 638-640. (in Chinese).

9. YangXY. PracticalChineseNewspaperreadabilityformula. Journalism Research. 1974; 30(13): 37-62. (inChinese).

10. Feng YL. The Use of Readability Measurement in Weibo News Reports--Take the "toxic capsule" report on Sina Weibo's "Headline News" as an example. Southeastern Propagation 2012; 8(11): 89-92. (in Chinese).

11. LI SS. A Survey of Studies on Readability. Journal of Pla University of Foreign Languages 2000; 4. 
12. Koo MM, Krass I, Aslani P. Factors influencing consumer use of written drug information. Ann Pharmacother 2003; 37(2): 259-67.

13. Vigilante JWJ, Wogalter JMS. The preferred order of over-the-counter (Otc) pharmaceutical label components. Drug Inf $J$ 1997; 31(3): 973-988..

14. Shrank WH, Agnew-Blais J, Choudhry NK, Wolf MS, Kesselheim AS, Avorn J, Shekelle P. The variability and quality of medication container labels. Arch Intern Med 2007; 167(16): 1760-5.

15. Grime J, Blenkinsopp A, Raynor DK, Pollock K, Knapp P. The role and value of written information for patients about individual medicines: a systematic review. Health Expect 2007; 10(3): 286-98.

16. Meng F, Li XL. Analysis of the Problems of Package Inserts of Traditional Chinese Medicine Injection. China Pharmacy 2011; 22(27): 2587-9. (in Chinese).

17. Gu Q, Zhang YQ, Xie GH. Research and analysis of 167 package inserts of chemical drug J. China Journal of Modern Medicine 2008; 18(10): 1443-1445. (in Chinese).

18. Ved JK. Package Inserts in India: Need for a Revision. International Journal of Pharma Sciences and Research 2010; 1(11): 454-6.

19. Ramdas D, Chakraborty A, Hs S, Faizan S, Kumar VP, Bn S. A study of package inserts in southern India. J Clin Diagn Res 2013;7(11): 2475-7.

20. Gupta VK, Pathak SS. Assessment of awareness and knowledge about package inserts amongst medical students: a questionnaire based study. IOSR J Pharm 2012; 2(2): 215-217.

21. Ng GQ, Sklar GE, Chng HT. An evaluation of the completeness of drug-drug interaction-related information in package inserts. Eur J Clin Pharmacol 2017; 73(2): 165-174.

22. Wolf MS, Davis TC, Shrank WH, Neuberger M, Parker RM. A critical review of FDA-approved medication guides. Patient Educ Couns 2006; 62(3): 316-22.

23. Singh KR, Munshi R, Arora D. Assessment of the degree of awareness among physicians and patients about drug package inserts. Int J Pharm Sci Res 2016; 7(3): 1338.

24. Pizzol TDSD, Moraes CG, Arrais PSD, Bertoldi AD, Ramos LR, Farias MR, Oliveira MA, Tavares NUL, Luiza VL, Mengue SS. Medicine package inserts from the users' perspective: are they read and understood? Rev Bras Epidemiol 2019; 22:e190009.

25. Pires $\mathrm{C}$,Vigário M,Cavaco A. Factors influencing subjects' comprehension of a set of medicine package inserts. Int J Clin Pharm 2016; 38(4): 888-98.

26. Zarea Gavgani V, Mirzadeh-Qasabeh S, Hanaee J, Hamishehkar H. Calculating reading ease score of patient package inserts in Iran. Drug Healthc Patient Saf 2018; 10: 9-19.

27. Shrank W, Avorn J, Rolon C, Shekelle P. Effect of content and format of prescription drug labels on readability, understanding, and medication use: A systematic review. Ann Pharmacother 2007; 41(5): 783-801.

28. Davis TC, Wolf MS, Bass PF 3rd, Thompson JA, Tilson $\mathrm{HH}$ Neuberger M, Parker RM. Literacy and misunderstanding prescription drug labels. Ann Intern Med 2006; 145(12): 887-94.

29. Sahm LJ, Wolf MS, Curtis LM, Behan R, Brennan M, Gallwey H, Mc Carthy S.What's in a label? An exploratory study of patient-centered drug instructions. Eur J Clin Pharmacol 2012; 68(5): 777-782 .

30. Fulton JA, Nelson LS. Medication labeling errors in non-Englishspeaking patients. Ann Pharmacother 2005; 39(2): 386-387.

31. Davis TC, Federman AD, Bass PF 3rd, Jackson RH, Middlebrooks M, Parker RM, Wolf MS. Improving Patient Understanding of Prescription Drug Label Instructions. J Gen Intern Med 2009; 24(1): 57-62

32. Dong SJ, Zhai SD. A summary of the design and practice of foreign patients' medication instructions. Chinese Drug Application and Monitoring 2013; (4): 227-231. (in Chinese).

33. Piñero-López MÁ, Modamio P, Lastra CF, Mariño EL. Readability Assessment of Package Inserts of Biological Medicinal Products from the European Medicines Agency Website. Drug Saf 2014; 37(7): 543-54..

34. Bernardini C, Ambrogi V, Fardella G, Perioli L, Grandolini G. How to improve the readability of the patient package leaflet: a survey on the use of colour, print size and layout. Pharmacol Res 2001; 43(5): 437-44.

35. Bernardini C, Ambrogi V, Perioli LC, Tiralti MC,Fardella G. Comprehensibility of the package leaflets of all medicinal products for human use: a questionnaire survey about the use of symbols and pictograms. Pharmacol Res 2000; 41(6): 679-88.

36. Pires C, Vigário M, Cavaco A. Graphical content of medicinal package inserts: an exploratory study to evaluate potential legibility issues. Health Info Libr J 2016; 33(2): 121-39.

37. Dowse R, Ramela T, Browne SH. An illustrated leaflet containing antiretroviral information targeted for low-literate readers: Development and evaluation. Patient Educ Couns 2011; 85(3): 508-15.

38. Katz MG, Kripalani S, Weiss BD. Use of pictorial aids in medication instructions: a review of the literature. Am J Health Syst Pharm 2006; 63(23): 2391-7.

39. Dowse R, Ehlers M. Medicine labels incorporating pictograms: do they influence understanding and adherence? Patient Educ Couns 2005; 58(1): 63-70.

40. Hämeen-Anttila K, Kemppainen K, Enlund H, Bush Patricia J, Marja A. Do pictograms improve children's understanding of medicine leaflet information? Patient Educ Couns 2004; 55(3): 371-8. 